1. Iijima, S. Helical microtubules of graphitic carbon. Nature 354, 56-58 (1991).

2. Ebbesen, T. W. \& Ajayan, P. M. Large scale synthesis of carbon nanotubes. Nature 358, 220-222 (1992).

3. De Heer, W. A., Chatelain, A. \& Ugarte, D. A carbon nanotube field-emission electron source. Science 270, 1179-1180 (1995)

4. Terrones, M. et al. Recent Advances in the Chemistry and Physics of Fullerenes and Related Materials Vol. 2 (eds Kadish, K. M. \& Ruoff, R. S.) 599-620 (Electrochem. Soc., Pennington, NJ, 1995).

5. Dai, H. J., Wong, E. W. \& Lieber, C. M. Probing electrical transport in nanomaterials: conductivity of individual carbon nanotubes. Science 272, 523-526 (1996).

6. Ebbesen, T. W. et al. Electrical conductivity of individual carbon nanotubes. Nature 382, 54-56 (1996).

. Tans, S. J. et al. Individual single-wall carbon nanotubes as quantum wires. Nature 386, 474-477 (1997).

8. Bockrath, M. et al. Single-electron transport in ropes of carbon nanotubes. Science 275, 1922-1925 (1997).

9. Thess, A. et al. Crystalline ropes of metallic carbon nanotubes. Science 273, $483-487$ (1996)

10. Treacy, M. M. J., Ebbesen, T. W. \& Gibson, J. M. Exceptionally high Young's modulus observed for individual nanotubes. Nature 381, 678-680 (1996).

11. Amelinckx, S. et al. A formation mechanism for catalytically grown helix-shaped graphite nanotubes. Science 265, 635-639 (1994).

12. Endo, M. et al. Pyrolytic carbon nanotubes from vapor-grown carbon fibres. Carbon 33, 873-881 (1995).

13. Hsu, W. K. et al. Condensed-phase nanotubes. Nature 377, 687 (1995).

14. Hsu, W. K. et al. Electrolytic formation of carbon nanostructures. Chem. Phys. Lett. 261, 161-166 (1996).

15. Thurston, J. T. et al. Cyanuric chloride derivatives I. Aminochloro-s-triazines. J. Am. Chem. Soc. 73, 2981-2983 (1951)

16. Chrisey, D. B. \& Hubler, G. K. (eds) Pulsed Laser Deposition of Thin Films (Wiley, New York, 1994).

17. Terrones, M. et al. Pyrolytically grown $\mathrm{B}_{\mathrm{x}} \mathrm{C}_{\mathrm{y}} \mathrm{N}_{\mathrm{z}}$ nanomaterials: nanofibres and nanotubes. Chem. Phys. Lett. 257, 576-582 (1996)

18. Li, W. Z. et al. Large synthesis of aligned carbon nanotubes. Science 274, 1701-1703 (1996).

19. Tennent, H. G., Barber, J. J. \& Hoch, R. US Patent No. 5578543 (1996).

20. Hausslein, R. W. Commercial manufacture and uses of carbon nanotubules. 187th Mtg of the Electrochem. Soc. (Abstr.) 175 (Electrochem. Soc., Pennington, NJ, 1995).

21. Niu, C., Sichel, E. K., Hoch, R., Moy, D. \& Tennent, H. High power electrochemical capacitors based on carbon nanotube electrodes. Appl. Phys. Lett. 70, 1480-1482 (1997).

Acknowledgements. We thank J. Thorpe, D. P. Randall, S. Tehuacanero, R. Hernández, P. Mexía, R. Guardián and L. Rendón for providing electron microscope facilities, and D. Bernaerts for discussions. We thank CONACYT-México (M.T. and H.T.), the ORS scheme for scholarships (M.T. and W.K.H.), DGAPA-UNAM IN 107-296 (H.T.), EU-TMR grant (J.O.), the Royal Society (London) and the EPSRC for financial support.

Correspondence and requests for materials should be addressed to D.R.M.W. (e-mail: d.walton@sussex. ac.uk).

\section{Chemical properties of element 106 (seaborgium)}

\section{Schädel ${ }^{\star}$, W. Brüchle ${ }^{\star}$, R. Dressler $\dagger$, B. Eichler $\dagger$, H. W. Gäggeler $\ddagger$, R. Günther $₫$, K. E. Gregorich $\|$, D. C. Hoffman $\|$, , S. Hübener\#, D. T. Jost $\dagger$, J. V. Kratz $\$$, W. Paulus $\$$, D. Schumann ${ }^{\text {t/ }}$, S. Timokhin ${ }^{\star *}$ N. Trautmann $\S$, A. Türler $\dagger$, G. Wirth ${ }^{\star}$ \& A. Yakuschev ${ }^{\star \star}$}

* Gesellschaft für Schwerionenforschung, 64291 Darmstadt, Germany $\dagger$ Labor für Radio- und Umweltchemie, Paul Scherrer Institut, 5232 Villigen, Switzerland

$\ddagger$ Department für Chemie und Biochemie, Universität Bern, 3012 Bern, Switzerland

\$Institute für Kernchemie, Universität Mainz, 55099 Mainz, Germany II Lawrence Berkeley National Laboratory, Berkeley, California 94720, USA 9 Glenn T. Seaborg Institute for Transactinium Science, Livermore,

California 94551, USA

\# Institut für Radiochemie, Forschungszentrum Rossendorf, 01314 Dresden, Germany

Institut für Analytische Chemie, Technische Universität Dresden, 01062 Dresden, Germany

** Flerov Laboratory of Nuclear Reactions, Joint Institute of Nuclear Research, Dubna, Russia

The synthesis, via nuclear fusion reactions, of elements heavier than the actinides, allows one to probe the limits of the periodic table as a means of classifying the elements. In particular, deviations in the periodicity of chemical properties for the heaviest elements are predicted as a consequence of increasingly strong relativistic effects on the electronic shell structure ${ }^{1-7}$. The trans- actinide elements have now been extended up to element 112 (ref. 8), but the chemical properties have been investigated only for the first two of the transactinide elements, 104 and 105 (refs 919). Those studies showed that relativistic effect render these two elements chemically different from their lighter homologues in the same columns of the periodic table (Fig. 1). Here we report the chemical separation of element 106 (seaborgium, Sg) and investigations of its chemical behaviour in the gas phase and in aqueous solution. The methods that we use are able to probe the reactivity of individual atoms, and based on the detection of just seven atoms of seaborgium we find that it exhibits properties characteristic of the group 6 homologues molybdenum and tungsten. Thus seaborgium appears to restore the trends of the periodic table disrupted by relativistic effects in elements 104 and 105 .

Calculations of the electron configurations of heavy atoms ${ }^{1-5}$ have predicted that sudden changes in the structure of the electron shells may appear due to strongly increasing relativistic effects. These relativistic effects are proportional to the square of the nuclear charge, which attracts electrons in spherically symmetric orbitals $(s$ and $\left.p_{1 / 2}\right)$ most strongly to the nucleus. This, in turn, means that the nuclear charge is more efficiently screened, thus allowing expansion of the non-spherical $d$ and $f$ orbitals. Because the chemical behaviour of an element is strongly dependent on the electronic configuration, such relativistic effects can lead to unexpected chemical properties $^{6,7}$.

Studies of the chemical properties of elements 104 (rutherfordium, Rf) and 105 (hahnium, Ha; the name dubnium has also been proposed, but has yet to be approved by the International Union of Pure and Applied Chemistry) were full of surprises ${ }^{18,19}$. The nontantalum-like behaviour of hahnium in aqueous solution ${ }^{10,11,20}$, for example, and its similarity to niobium and/or protactinium, depending on its chemical environment, demonstrated that the chemical properties cannot be reliably extrapolated from the trends observed in its lighter homologues. Such surprises have also been seen in thermo-chromatographic ${ }^{9,21}$ and gas-chromatographic experiments ${ }^{12,13,15,16}$. Therefore, it is of interest to investigate whether the chemical properties of element 106 (seaborgium, Sg) resemble those of the lighter homologues in group 6 (molybdenum and tungsten) or those of the pseudo-group-6 element uranium.

We synthesized the most neutron-rich seaborgium isotopes ${ }^{22,23}$, ${ }^{265} \mathrm{Sg}$ and ${ }^{266} \mathrm{Sg}$, in a nuclear fusion reaction between ${ }^{22} \mathrm{Ne}$ ions from the GSI UNILAC accelerator and $\mathrm{a}^{248} \mathrm{Cm}$ target with a rate of the order of one atom per hour (ref. 24). The ${ }^{265} \mathrm{Sg}$ and ${ }^{266} \mathrm{Sg}$ nuclei were knocked out of the target foil and were stopped in helium gas loaded with tiny $(0.1-1 \mu \mathrm{m})$ solid particles (aerosols). Within about three seconds, the helium transported the reaction products-attached to aerosols - along capillary tubes to two different sets of chemical devices. To provide conclusive evidence that a seaborgium atom had passed through the chemical separation procedures, the experiments were designed to detect the characteristic $\alpha$-decay chains of the isotope ${ }^{265} \mathrm{Sg}$, and the corresponding daughter nuclides ${ }^{261} \mathrm{Rf}$ and ${ }^{257} \mathrm{No}$ (ref. 24). In addition, fission fragments were measured from spontaneous fission decay, which would arise from ${ }^{262} \mathrm{Rf}$, the $\alpha$-decay product of ${ }^{266} \mathrm{Sg}$. An earlier attempt to perform a chemical separation of element 106 fell short of unambiguously showing that the observed, by itself unspecific, spontaneous fission decay originated from an isotope of element 106 (ref. 25). We have applied two chemical separation techniques, one probing the formation of volatile oxychlorides in a gas-chromatographic experiment, and another one probing the formation of oxo- or oxyfluoride complexes in aqueous solution by liquid chromatography.

In classical gas chromatography, a substance under investigation is introduced into a flowing stream, and the time taken for the sample to emerge from chromatographic system is measured. In our experiment, in contrast, the nuclear reaction products were continuously supplied and separated in OLGA III-the on-line gas chemistry apparatus ${ }^{17}$. This technique uses the half-life of the 


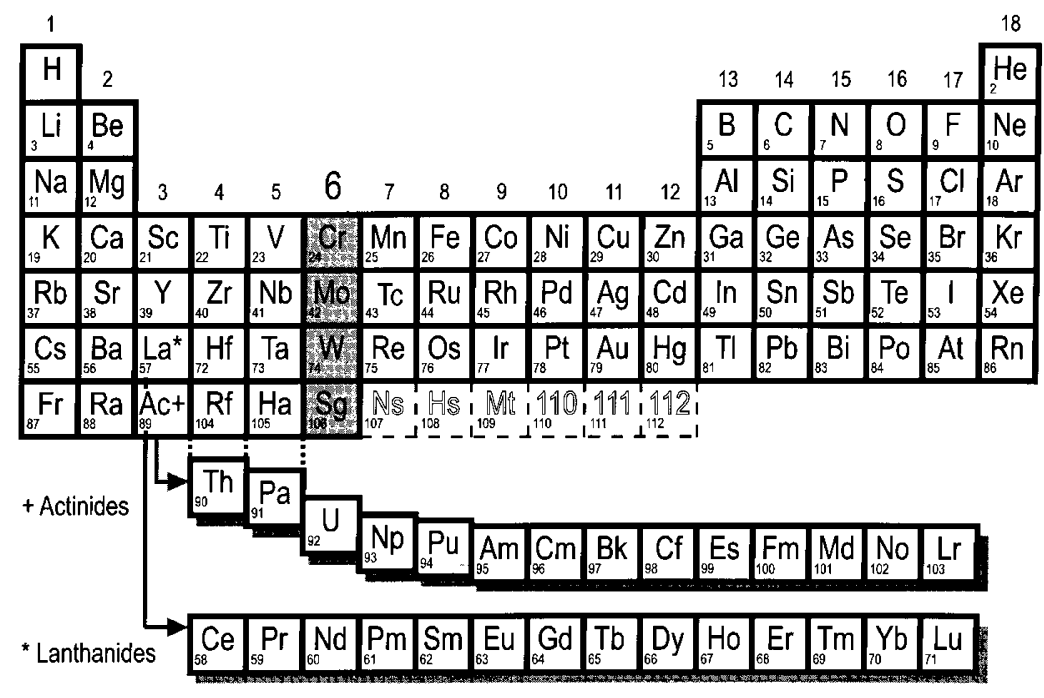

Figure 1 Periodic table of the elements. The arrangement of the actinides reflects the fact that the first actinide elements still resemble, to a decreasing extent, the chemistry of the other groups: Th the fourth group below $\mathrm{Hf}, \mathrm{Pa}$ the fifth group below $\mathrm{Ta}$, and $U$ the sixth group below $W$. The known transactinide elements 104 to 112 take the positions from below $\mathrm{Hf}$ in group 4 to below $\mathrm{Hg}$ in group 12. Element 106, seaborgium $(\mathrm{Sg})$, the heaviest element chemically investigated, is placed in group 6 .

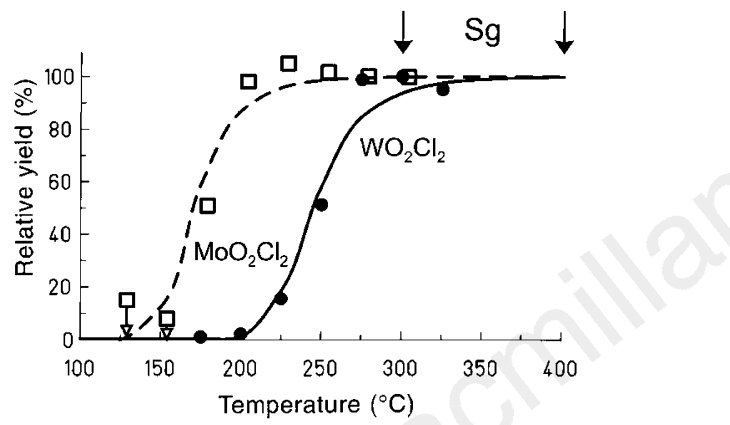

Figure 2 Measured relative yields of the compounds $\mathrm{MoO}_{2} \mathrm{Cl}_{2}$ (open squares) and $\mathrm{WO}_{2} \mathrm{Cl}_{2}$ (filled circles) versus temperature of the gas-chromatographic column. The lines are the result of Monte Carlo simulations which model the behaviour of the compounds during separation. Arrows indicate the temperatures at which seaborgium was separated.

nuclide under investigation as an 'internal' clock for the chromatographic process. Just half of the atoms introduced into the gaschromatographic column will emerge from the other end when the time taken for an atom to pass through the column (retention time) corresponds to the nuclide's half-life. In this way, yields (that is, the ratio of the quantity introduced into the column to the quantity detected after the column) can be converted into retention times when the column is run at various temperatures. Figure 2 shows the behaviour of compounds of the short-lived isotopes of the homologues molybdenum and tungsten under the experimental conditions used for the separation of seaborgium. Reactive gaseschlorine saturated with thionyl chloride, and a little oxygenwere added to the chemically inert carrier-gas helium. Given a sufficiently high temperature, the volatile oxychloride compounds form. Thermodynamic calculations indicate the formation of dioxydichlorides as the most stable compond. Figure 2 shows that $\mathrm{MoO}_{2} \mathrm{Cl}_{2}$ is more volatile than $\mathrm{WO}_{2} \mathrm{Cl}_{2}$ because the former passes through the chromatography column at a lower temperature. The experiment with seaborgium atoms was carried out at temperatures of 300 and $400{ }^{\circ} \mathrm{C}$, high enough for detection of the volatile oxychlorides-assuming that these seaborgium compounds do not exhibit unexpectedly low volatilities.

In the course of the OLGA experiment, the chemically separated volatile compounds leaving the end of the quartz gas-chromatography column are deposited on aerosol particles and rapidly transported in a gas stream to a detector. Here, about six seconds after their formation, the seaborgium compounds are deposited on thin foils, mounted on a revolving wheel. The wheel is rotated in a ten-second cycle so as to position the samples between detector pairs, which detect the $\alpha$-particles and spontaneous-fission fragments, and measure their characteristic energies and time sequences. With OLGA III, it was possible for the first time to detect unambiguously three $\alpha$-decay chains of ${ }^{265} \mathrm{Sg}$ after solid/gasphase chemical separation (Fig. 3). An additional $\alpha$-decay, which was followed 2.8 seconds later by a spontaneous fission, can be assigned to the decay of the neighbouring isotope ${ }^{266} \mathrm{Sg}$.

Our chemical results show that, under the given conditions, element 106 forms a volatile oxychloride at 300 and $400^{\circ} \mathrm{C}$. At each temperature, two $\alpha$-decay chains were observed. Thermodynamic calculations indicate that $\mathrm{SgO}_{2} \mathrm{Cl}_{2}$ is formed, in analogy to $\mathrm{MoO}_{2} \mathrm{Cl}_{2}$ and $\mathrm{WO}_{2} \mathrm{Cl}_{2}$. This agrees with the expected behaviour from an extrapolation in group 6 of the periodic table and with theoretical calculations ${ }^{7}$.

Further chromatographic separations were carried out with single seaborgium atoms to determine its ion valency and complex formation in aqueous solution ${ }^{24}$. Using the separation apparatus $\mathrm{ARCA}^{26}$ (automated rapid chemistry apparatus), investigations with molybdenum and tungsten showed that hexavalent ions could be eluted from cation exchange columns with dilute nitric/ hydrofluoric acid. This chemical system makes use of characteristic differences in the formation of cationic, neutral and anionic complexes with $\mathrm{F}^{-}$ions between elements of groups 3, 4, 5 and 6. Di- and trivalent actinides, group 4 elements, and the pseudo-group-6 element uranium, present as $\mathrm{UO}_{2}^{2+}$, are retained on the cation exchange resin. However, the group 6 elements molybdenum and tungsten form anions of the type $\mathrm{MO}_{4}^{2-}, \mathrm{MO}_{3} \mathrm{~F}^{-}$or $\mathrm{MO}_{2} \mathrm{~F}_{3}^{-}$(where $\mathrm{M}$ indicates a metal ion $)^{27}$. Moreover, the formation of a neutral compound like $\mathrm{MO}_{2} \mathrm{~F}_{2}$ can not be excluded. Anionic and neutral species readily elute from the column.

Theoretical calculations of the electronic structure and redox potentials predict that, within the series of $\mathrm{MO}_{4}^{2-}$ ions formed in aqueous solution, $\mathrm{SgO}_{4}^{2-}$ will be the most stable ${ }^{28}$. From this, we expect that in very dilute hydrofluoric acid seaborgium forms $\mathrm{SgO}_{4}^{2-}$ which should follow the lighter homologues molybdenum and tungsten in the chemical separation.

During the course of more than 5,000 liquid-chromatographic separations (elution time, 10 seconds each), it was possible to detect three $\alpha$-decay chains of the daughter nuclei of ${ }^{265} \mathrm{Sg}$-namely, the $\alpha$-decay of ${ }^{261} \mathrm{Rf}$ and ${ }^{257} \mathrm{No}$ - in the seaborgium fraction ${ }^{24}$ (Fig. 3). They constitute unambiguous proof that seaborgium has passed 


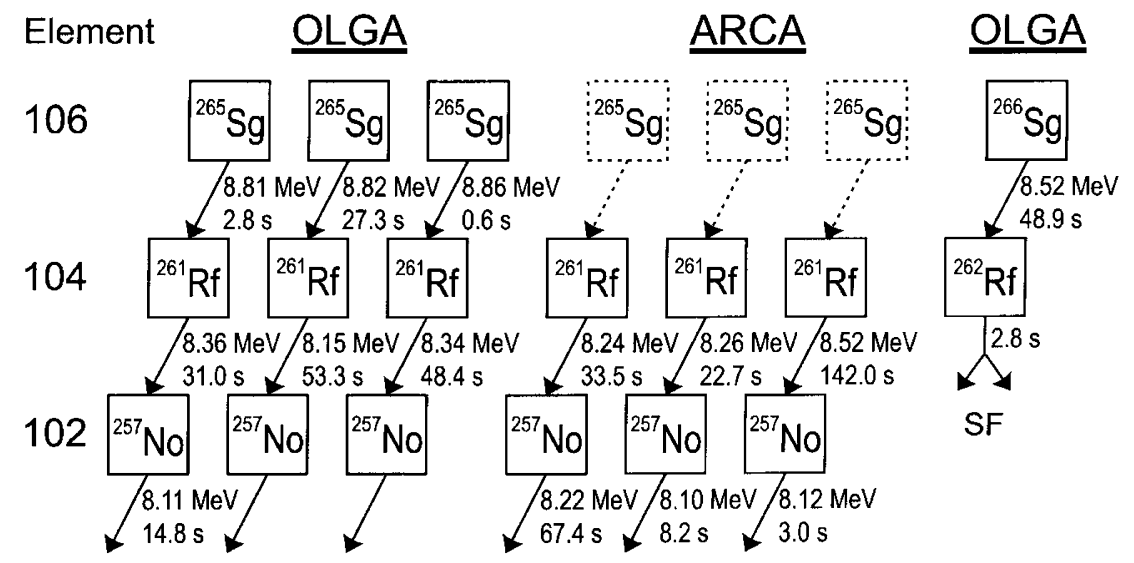

Figure 3 The observed nuclear decay chains from the seaborgium isotopes ${ }^{265} \mathrm{Sg}$ and ${ }^{266} \mathrm{Sg}$, which allowed an unambiguous identification of seaborgium after chemical separation with ARCA (automated rapid chemistry apparatus) and OLGA (on-line gas chemistry apparatus). The $\alpha$-decay energies are given in $\mathrm{MeV}$, and the observed life-times in seconds.

through the column, given that both of these nuclides could only be present in the seaborgium fraction due to the $\alpha$-decay of ${ }^{265} \mathrm{Sg}$. Isotopes of the elements 104 and 102 formed directly or from decay of seaborgium before chemical separation were chemically separated. With a $90 \%$ probability, the parent nuclei had already decayed into the daughter nucleus ${ }^{261} \mathrm{Rf}$ in the time between the end of chemical separation and the commencement of measurement about 28 seconds later. This is very likely in view of the short life-times measured for the ${ }^{265} \mathrm{Sg}$ decays with OLGA (Fig. 3).

The first liquid-chromatographic separation of element 106 shows that at least a substantial fraction of the formed seaborgium behaves similarly to its lighter homologues molybdenum and tungsten, that is, that its behaviour is typical of a hexavalent ion belonging to group 6 of the periodic table. Presumably, seaborgium forms $\mathrm{SgO}_{4}^{2-}$ or a neutral complex.

Both our isothermal gas chromatographic and liquid chromatographic separations clearly indicate that seaborgium behaves similarly to its lighter homologues molybdenum and tungsten, and its behaviour is typical for a group 6 element of the period table. These results support the assumption that the chemistry of elements 107 to 112 will be homologous to that of the group 7 to 12 elements rhenium to mercury-if increasingly strong relativistic effects do not alter the chemical properties to such an extent that they are no longer predictable from such simple extrapolations, as seen for elements 104 and 105.

Received 27 January; accepted 12 May 1997.

1. Fricke, B. \& Greiner, W. On the chemistry of superheavy elements around $Z=164$. Phys. Lett. B 30 317-319 (1969).

2. Desclaux, J.-P. \& Fricke, B. Relativistic prediction of the ground state of atomic lawrencium. J. Phys. 41, 943-946 (1980)

3. Glebov, V. A., Kasztura, L., Nefedov, V. S. \& Zhuikov, B. L. Is element 104 (kurchatovium) a pelement? II. Relativistic calculations of the electronic atomic structure. Radiochim. Acta 46, 117-121 (1989).

4. Johnson, E., Fricke, B., Keller, O. L., Nestor, C. W. \& Tucker, T. C. Ionization potentials and radii of atoms and ions of element 104 (unnilquadium) and of hafnium $(2+)$ derived from multiconfiguration Dirac-Fock calculations. J. Chem. Phys. 93, 8041-8050 (1990).

5. Eliav, E., Kaldor, U. \& Ishikawa, Y. Ground state electron configuration of rutherfordium: role of dynamic correlation. Phys. Rev. Lett. 74, 1079-1082 (1995).

6. Pyykkö, P. Relativistic effects in structural chemistry. Chem. Rev. 88, 563-594 (1988).

7. Pershina, V. G. Electronic structure and properties of the transactinides and their compounds. Chem. Rev. 96, 1977-2101 (1996).

8. Hofmann, S. et al. The new element 112. Z. Phys. A 354, 229-230 (1996).

9. Zvara, I., Belov, V. Z., Domanov, V. P. \& Shalaevskii, M. R. Chemical isolation of nilsbohrium as ekatantalum in the form of the anhydrous bromide. II. Experiments with a spontaneously fissioning isotope of nilsbohrium. Sov. Radiochem. 18, 328-334 (1976).

10. Gregorich, K. E. et al. Aqueous chemistry of element 105. Radiochim. Acta 43, 223-231 (1988).

11. Kratz, J. V. et al. Chemical properties of element 105 in aqueous solution: halide complex formation and anion exchange into triisooctyl amine. Radiochim. Acta 48, 121-133 (1989).

12. Gäggeler, H. W. et al. Gas phase chromatography experiments with bromides of tantalum and element 105. Radiochim. Acta 57, 93-100 (1992).

13. Gäggeler, H. W. On-line gas chemistry experiments with transactinide elements. J. Radioanal. Nucl. Chem. 183, 261-271 (1994)

14. Czerwinski, K. R. et al. Solution chemistry of element 104: Part I. Liquid-liquid extractions with triisoctylamine. Radiochim. Acta 64, 23-28 (1994); Solution chemistry of element 104: Part II. Liquid-liquid extractions with tributylphosphate. Radiochim. Acta 64, 29-35 (1994).

15. Türler, A. et al. On-line gas phase chromatography with chlorides of niobium and hahnium (element 105). Radiochim. Acta 73, 55-66 (1996).
16. Kadkhodayan, B. et al. On-line gas chromatographic studies of chlorides of rutherfordium and homologs $\mathrm{Zr}$ and Hf. Radiochim. Acta 72, 169-178 (1996).

17. Türler, A. Gas phase chemistry experiments with transactinide elements. Radiochim. Acta 72, 7-17 (1996).

18. Schädel, M. Chemistry of the transactinide elements. Radiochim. Acta 70/71, 207-223 (1996)

19. Hoffman, D. C. Chemistry of the heaviest elements. Radiochim. Acta 72, 1-6 (1996).

20. Zimmermann, H. P. et al. Chemical properties of element 105 in aqueous solution: back extraction from triisooctyl amide into $0.5 \mathrm{M} \mathrm{HCl}$. Radiochim. Acta 60, 11-16 (1993).

21. Zvara, I. et al. Gas chromatography and thermochromatography in the study of transuranium elements. Sov. Radiochem. 16, 709-715 (1974).

22. Lougheed, R. W. et al. in Proc. Int. Conf. Actinides-93 (eds Clark, D. L., Hobart, D. E. \& Fuger, F.) 161 (Elsevier, Amsterdam, 1994); J. Alloy Compounds 213/214, 61-66 (1994).

23. Lazarev, Yu. A. et al. Discovery of enhanced nuclear stability near the deformed shells $\mathrm{N}=162$ and $\mathrm{Z}=$ 108. Phys. Rev. Lett. 73, 624-627 (1994)

24. Schädel, M. et al. First aqueous chemistry with seaborgium (element 106). Radiochim. Acta (in the press)

25. Timokhin, S. N., Yakushev, A. B., Honggui Xu, Perelygin, V. P. \& Zvara, I. Chemical identification of element 106 by thermochromatography. J. Radioanal. Nucl. Chem. Lett. 212, 31-34 (1996).

26. Schädel, M. et al. ARCA II - a new apparatus for fast, repetitive HPLC separations. Radiochim. Acta 48, 171-176 (1989)

27. Tytko, K.-H. \& Gras, D. in Gmelin, Handbook of Inorganic Chemistry, Molybdenum Suppl. Vol. B 3b (Springer, Heidelberg, 1989).

28. Pershina, V. \& Fricke, B. The electronic structure of the group 6 oxyanions $\left[\mathrm{MO}_{4}\right]^{2-}$, where $\mathrm{M}=\mathrm{Cr}$, Mo, W, and element 106. Radiochim. Acta 65, 13-17 (1994).

Acknowledgements. We are indebted to the Division of Chemical Sciences, Office of Basic Energy Research, US Department of Energy, for making the ${ }^{248} \mathrm{Cm}$ target material through the transplutonium element production program at the Oak Ridge National Laboratory. We also thank the staff and crew of the GSI UNILAC, the TRIGA Mainz reactor, the Philips-cyclotron at PSI, and the MPI Heidelberg tandem accelerator for their help. This work was supported in part by the Bundesministerium für Bildung, Wissenschaft, Forschung und Technologie (BMBF), the Swiss National Science Foundation, and the Chemical Sciences Division of the Office of Basic Energy Sciences, US Department of Energy.

Correspondence should be addressed to M.S. (e-mail: m.schaedel@gsi.de).

\section{Vegetation and climate change in northwest America during the past $125 \mathrm{kyr}$}

\section{Cathy Whitlock \& Patrick J. Bartlein}

Department of Geography, University of Oregon, Eugene, Oregon 97403, USA

Vegetation records spanning the past $21 \mathrm{kyr}$ in western North America display spatial patterns of change that reflect the influence of variations in the large-scale controls of climate ${ }^{1}$. Among these controls are millennial-scale variations in the seasonal cycle of insolation and the size of the ice sheet, which affect regional climates directly through changes in temperature and net radiation, and indirectly by shifting atmospheric circulation. Longer vegetation records provide an opportunity to examine the regional response to different combinations of these large-scale controls, and whether non-climatic controls are important. But most of the longer North American records ${ }^{2,3}$ are of insufficient quality to allow a robust test, and the long European records $s^{4-9}$ are in regions where the vegetation response to climate is often 